\title{
Decentralized Controller Robustness Improvement Using Longitudinal Overlapping Decomposition - Application to Web Winding System
}

\author{
Doghmane Mohamed Zinelabidine ${ }^{1,2}$, Kidouche Madjid ${ }^{1}$ \\ ${ }^{1}$ Laboratory of Applied Automatic, University M'hamed Bougara, \\ Avenue $1^{\text {er }}$ Novembre 35000, Boumerdes \\ ${ }^{2}$ Exploration-Production Division, \\ Sonatrach, Algeria \\ mdoghmane@for.hmd.sonatrach.dz
}

\begin{abstract}
Most of industrial systems are described by complex mathematical models with high order dimensions, wherein control engineering is facing big challenges in designing robust controllers for such type of systems; the main difficulty lies in founding a control structure that is tractable for large scale systems without complicating the controller design from one side, and with preserving controller robustness from the other side. Our study is focused on designing robust controller for industrial systems with complex structure. The robustness of decentralized controller has been improved by using overlapping decomposition approach; the obtained controller has been applied to Web Winding system with longitudinal structure. The designed approach leads to more computational technique, thus to easy-implementable controllers. Moreover, simulation results are presented with different input signals so that the usefulness of the proposed approach can be generalized.
\end{abstract}

Index Terms-Control engineering; Large scale systems; Overlapping decomposition; Robust control.

\section{INTRODUCTION}

Many industrial technologies use web winding system for different tasks such as transporting products and materials [1]. The outputs of such system are web tensions and web velocities; and the inputs of the controlled system are the maximum velocity of the web with predefined values of tensions. The Mathematical model derivation of this system leads to dimensional state space representation [2], which requires huge amount of computational effort to analyse dynamic process. Thus, the control design becomes more complicated. These complications require the use of decomposition strategies to divide the analysis and control problem of the original system into lower subsystems. Subcontrollers can be designed separately for each subsystem, and then the sub-solutions can be jointed together with respect to interconnection constraints to provide global solution of the original system.

The usefulness of decomposition strategies for web

Manuscript received 2 February, 2018; accepted 20 June, 2018. winding system has been proved in many papers ([2], [3], [4], and [5]), in [6] multivariable control decoupling has been used in cold strip mill to decompose tensions and speed, however, the controller design is realized based on invariance principle with consideration that interconnection constraints between velocities and tensions are static, thus strong dynamic of interconnection has not been considered. Control complexity of web winding system has been analysed by few researchers, Stankovic el al. [7] proposed a general overlapping controller that deals with tracking inputs variations with the assumption that the constraints are known and given, Hou et al. [8] proposed used linear matrix inequalities (LMIs) approach for decentralized control design to minimize variations in web tension, however the disturbances in web velocity has not be considered.

Decomposition strategies are developed to reduce system model dimension as well as simplification controller implementation, one of the best and direct applications for the recent development in decomposition strategies and control design is web winding system [4]. Draudvilien and Mazeika [9] investigated the effect of spectrum decomposition on system response and found that the systematic errors can be generated by modelling approach and decomposition technique as well. Feng et al. [10] used decomposition strategy in order to design $H_{\infty}$ controller for systems with uncertainties and time-delay in model dynamic. However, they assumed that disturbance has low frequency values which is acceptable for vehicle platoon system but not for web winding systems. Traditional control strategies based on PID controller do not achieve good decoupling especially for flexible webs. Moreover, robust multivariable controllers, recently proposed for this application ([1], [3], and [8]), are only powerful for reduced size systems. Robust decentralized overlapping output feedback control strategy is proposed in [11] for large scale systems; the load frequency controller designed with the presence of generation rate constraints and time varying delays, it guarantee the stability of the closed-loop system, the effectiveness of this approach is demonstrated through 
simulation results of a multi-area interconnected power system [11], it is an innovative strategy for winding systems, it is based on large scale control strategies without the inconvenient of having too many inputs and outputs for one controller [12].

Our work aims to improve decentralized controller robustness using overlapping decomposition applied to web winding system model; this strategy is based on decoupling the systems' model into lower interconnected subsystems that can be stabilized locally, and then conclude about global stability.

\section{DESCRIPTION OF SYSTEM}

Web winding system is quite common in industry [13]; it is basically composed of: unwinding, traction and winding motors. Inputs and outputs of this multivariable system (Fig. 1) are

- Inputs are control signals references $T_{u}^{*}, v_{3}^{*}$ and $T_{w}^{*}$.

- Outputs are, respectively, winding and unwinding web tensions $T_{w}, T_{u}$ and the web velocity $v_{3}$.

The web velocity is imposed by the traction motor whereas, the web tensions are controlled by the unwinding and winding motors. The mathematical model of web winding system is taken from [2].Wherein, for the $i^{\text {th }}$ order of the model, we have

$$
L_{i-1} \dot{T}_{i}=E S\left(v_{i}-v_{i-1}\right)+T_{i-1} v_{i-1}-T_{i} v_{i},
$$

where $E$ is young modulus of elasticity, and $S$ is surface area. In this study, we consider that the length between two successive motors is $L_{i-1}=L_{i}=L_{i+1}=L$, this implies that

$$
\dot{T}_{i}=\frac{E S}{L}\left[-v_{i-1}\left(E S+T_{i-1}\right)+v_{i}\left(E S-T_{i}\right)\right] .
$$

According to the web winding dynamic, we can assume that $-v_{i-1}\left(E S+T_{i-1}\right) \simeq-v_{1}\left(E S+T_{1}\right)$, so that (2), becomes

$$
\dot{T}_{i}=\frac{E S}{L}\left[-v_{1}\left(E S+T_{1}\right)+v_{i}\left(E S-T_{i}\right)\right] .
$$

For the velocities equation, we have

$$
\frac{d}{d t}\left(J_{i} \Omega_{i}\right)=R_{i}\left(T_{i+1}-T_{i}\right)+C_{e m}+C_{f} .
$$

The angular velocity is $\Omega_{i}=v_{i} / R_{i}$, and friction coefficient is $C_{f}=-f_{i}(t) \Omega_{i}, f$ is nonlinear function, this implies that

$$
\dot{v}_{i}=\frac{R_{i}}{J_{i}}\left[R_{i}\left(T_{i+1}-T_{i}\right)+\operatorname{Cem}_{i}-f_{i}(t) \frac{v_{i}}{R_{i}}\right] .
$$

From (3), and (5), we can obtain the system below:

$$
\left\{\begin{array}{l}
\dot{\mathbf{X}}=\mathbf{A} \times \mathbf{X}+\mathbf{B} \times \mathbf{U} \\
\mathbf{Y}=\mathbf{C} \times \mathbf{X}
\end{array}\right.
$$

where $\mathbf{X}^{T}=\left[\begin{array}{lllllllll}v_{1} & T_{2} & v_{2} & T_{3} & v_{3} & T_{4} & v_{4} & T_{5} & v_{5}\end{array}\right]$ and $\mathbf{Y}^{T}=\left[\begin{array}{lll}T_{u} & v_{3} & T_{w}\end{array}\right] ; T_{1}=0$ and $f$ is linearized around operating point, with $T_{u}=\frac{T_{2}+T_{3}}{2}, T_{w}=\frac{T_{4}+T_{5}}{2}$, and $\mathbf{U}^{T}=\left[\begin{array}{lllllllll}\mathrm{Cem}_{1} & 0 & \mathrm{Cem}_{2} & 0 & \mathrm{Cem}_{3} & 0 & \mathrm{Cem}_{4} & 0 & \mathrm{Cem}_{5}\end{array}\right]$ $\mathbf{A}(t), \mathbf{B}(t)$, and $\mathbf{C}(t)$ are time varying matrices [10] which have an overlapping structures: $\mathbf{A}(t)=\left[\begin{array}{lll}A_{11} & A_{12} & A_{13} \\ A_{21} & A_{22} & A_{23} \\ A_{31} & A_{32} & A_{33}\end{array}\right]$, $\mathbf{B}(t)=\left[\begin{array}{lll}B_{11} & B_{12} & B_{13} \\ B_{21} & B_{22} & B_{23} \\ B_{31} & B_{32} & B_{33}\end{array}\right], \mathbf{C}=\left[\begin{array}{lll}C_{11} & C_{12} & C_{13} \\ C_{21} & C_{22} & C_{23} \\ C_{31} & C_{32} & C_{33}\end{array}\right]$.

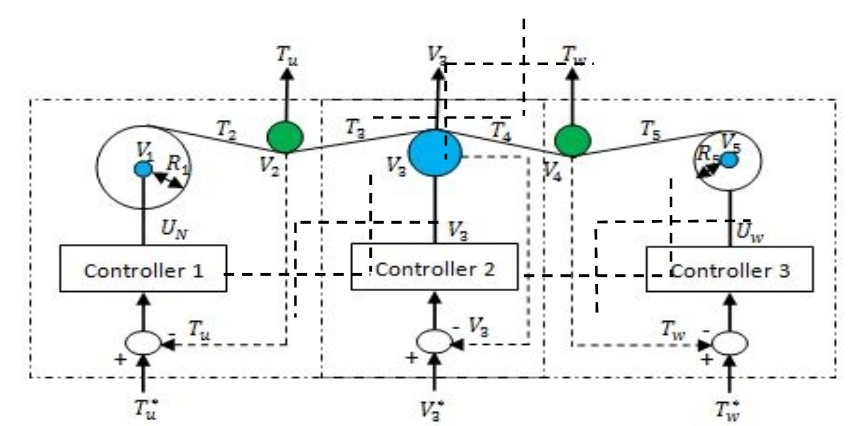

Fig. 1. Schema of three-motor web winding system.

Figure 2 shows Simulink block for the closed loop web winding system without applying any control strategy.

\section{DECOMPOSITION CONCEPT}

Decomposition techniques are widely introduced in several works in order to reduce the control design complexity of large scale systems ([14]). The system given by (6) can be expanded into larger system then decomposed into simpler subsystems; using transformation matrices $\mathbf{T}$ and $\mathbf{G}$ we can expand inputs, states and outputs of state space representation of web winding system.

The matrices $\mathbf{T}$ and $\mathbf{G}$ are chosen so that:

$$
\left\{\begin{array}{l}
\mathbf{X}_{e}=\mathbf{T} \times \mathbf{X} \\
\mathbf{U}_{e}=\mathbf{G} \times \mathbf{U} \\
\mathbf{Y}_{e}=\mathbf{G} \times \mathbf{Y}
\end{array}\right.
$$

which implies that:

$$
\left\{\begin{array}{l}
\mathbf{X}=\mathbf{T}^{I} \times \mathbf{X}_{e} \\
\mathbf{U}=\mathbf{G}^{I} \times \mathbf{U}_{e} \\
\mathbf{Y}=\mathbf{G}^{I} \times \mathbf{Y}_{e}
\end{array}\right.
$$

and: 


$$
\left\{\begin{array}{l}
\dot{\mathbf{X}}=\mathbf{T}^{I} \times \dot{\mathbf{X}}_{e} \\
\mathbf{U}=\mathbf{G}^{I} \times \mathbf{U}_{e} \\
\mathbf{Y}=\mathbf{G}^{I} \times \mathbf{Y}_{e}
\end{array}\right.
$$

By replacing (8) and (9) in (6), we can find:

$$
\left\{\begin{array}{l}
\mathbf{T}^{I} \times \dot{\mathbf{X}}_{e}=\mathbf{A} \times \mathbf{T}^{I} \times \mathbf{X}_{e}+\mathbf{B} \times \mathbf{G}^{I} \times \mathbf{U}_{e} \\
\mathbf{G}^{I} \times \mathbf{Y}_{e}=\mathbf{C} \times \mathbf{T}^{I} \times \mathbf{X}_{e}
\end{array}\right.
$$

Multiplying parts of the last equation by $\mathbf{T}$ and $\mathbf{G}$ respectively, we find:

$$
\left\{\begin{array}{l}
\dot{\mathbf{X}}_{e}=\mathbf{T} \times \mathbf{A} \times \mathbf{T}^{I} \times \mathbf{X}_{e}+\mathbf{T} \times \mathbf{B} \times \mathbf{G}^{I} \times \mathbf{U}_{e}, \\
\mathbf{Y}_{e}=\mathbf{G} \times \mathbf{C} \times \mathbf{T}^{I} \times \mathbf{X}_{e}
\end{array}\right.
$$

Using the pairs $\left(\mathbf{X}_{e}, \mathbf{U}_{e}, \mathbf{Y}_{e}\right)$, the expanded system can be written as:

$$
\left\{\begin{array}{l}
\dot{\mathbf{X}}_{e}=\mathbf{A}_{\mathbf{e}} \times \mathbf{X}_{e}+\mathbf{B}_{e} \times \mathbf{U}_{e} \\
\mathbf{Y}_{e}=\mathbf{C}_{e} \times \mathbf{X}_{e}
\end{array}\right.
$$

By comparing (11), with (12), we can find expansion conditions for linearized web winding system in the open loop form:

$$
\left\{\begin{array}{l}
\mathbf{A}_{\mathbf{e}}=\mathbf{T} \times \mathbf{A} \times \mathbf{T}^{\mathbf{I}}, \\
\mathbf{B}_{e}=\mathbf{T} \times \mathbf{B} \times \mathbf{G}^{\mathbf{I}}, \\
\mathbf{C}_{e}=\mathbf{G} \times \mathbf{C} \times \mathbf{T}^{\mathbf{I}},
\end{array}\right.
$$

or:

$$
\left\{\begin{array}{l}
\mathbf{A}=\mathbf{T}^{\mathbf{I}} \times \mathbf{A} \times \mathbf{T} \\
\mathbf{B}=\mathbf{T}^{\mathbf{I}} \times \mathbf{B}_{e} \times \mathbf{G} \\
\mathbf{C}=\mathbf{G}^{\mathbf{I}} \times \mathbf{C}_{e} \times \mathbf{T}
\end{array}\right.
$$

The system (12) is an expansion of the system (6) (or system (6) is contraction of system (12)) if there exist transformation matrices $\mathbf{T}$ and $\mathbf{G}$ satisfying (13) and (14) such that for any initial states $\left(\mathbf{X}_{e}(0), \dot{\mathbf{X}}_{e}(0)\right)$ and for any input $U_{e}(t) \in R^{m_{e}}$ for all $t \geq 0$ we have [15].

$\left\{\begin{array}{l}\mathbf{X}_{e}(0)=\mathbf{T X}(0), \\ \dot{\mathbf{X}}_{e}(0)=\mathbf{T} \dot{\mathbf{X}}(0), \\ \mathbf{U}_{e}(t)=\mathbf{G} \mathbf{U}(t),\end{array} \Rightarrow\left\{\begin{array}{l}\mathbf{X}_{e}(t)=\mathbf{T X}(t), \\ \dot{\mathbf{X}}_{e}(t)=\mathbf{T} \dot{\mathbf{X}}(t) .\end{array}\right.\right.$

Consider the transformation T, G satisfying (15) for the original system; we define the transform matrices $\mathbf{T}_{\mathbf{d}}$ and $\mathbf{C}_{\mathbf{d}}$ for the expanded system such that:

$$
\left\{\begin{array} { l } 
{ \mathbf { X } ( 0 ) = \mathbf { T } ^ { \mathbf { I } } \mathbf { X } _ { e } ( 0 ) } \\
{ \mathbf { U } ( t ) = \mathbf { G } ^ { \mathbf { I } } \mathbf { U } _ { e } ( t ) }
\end{array} \Rightarrow \left\{\begin{array}{c}
\mathbf{X}_{e d}\left(t_{i}\right)=\mathbf{T}_{\mathbf{d}} \mathbf{X}_{d}\left(t_{i}\right) \\
\mathbf{y}_{e}\left(t_{i}\right)=\mathbf{C}_{\mathbf{d}} \mathbf{x}_{d}\left(t_{i}\right)
\end{array}\right.\right.
$$

The system (12) is found by applying expansion principle, it can be decomposed using overlapping technique [15] as it is explained in section four.

\section{OVERLAPPING DECOMPOSITION THEORIES}

In this section, we discuss the overlapping decomposition for closed loop system under an output feedback controller, consider the output feedback controller $\mathbf{U}=\mathbf{K} \times \mathbf{Y}+\mathbf{w}$, where $\mathbf{w}^{T}=\left[\begin{array}{ccc}T_{u}^{*} & v_{3}^{*} & T_{w}^{*}\end{array}\right]$ are external input signals.



Fig. 2. Centralized output feedback controller of overlapped web winding system without decomposition. 
If we replace $U$ by its value in the open loop overlapped system given by (6), the system becomes:

$$
\left\{\begin{array}{l}
\dot{\mathbf{X}}=[\mathbf{A}+\mathbf{B} \times \mathbf{K} \times \mathbf{C}] \mathbf{X}+\mathbf{B} \times \mathbf{w} \\
\mathbf{Y}=\mathbf{C} \times \mathbf{X}
\end{array}\right.
$$

The closed loop system given by (17) can be expanded into larger non overlapped system:

$$
\left\{\begin{array}{l}
\dot{\mathbf{X}}_{e}=\left[\mathbf{A}_{e}+\mathbf{B}_{e} \times \mathbf{K}_{e} \times \mathbf{C}_{e}\right] \mathbf{X}_{e}+\mathbf{B}_{e} \times \mathbf{w}_{e} \\
\mathbf{Y}_{\mathbf{e}}=\mathbf{C}_{e} \times \mathbf{X}_{e}
\end{array}\right.
$$

if and only if:

$$
\left\{\begin{array}{l}
\mathbf{A}_{\mathbf{e}}=\mathbf{T} \times \mathbf{A} \times \mathbf{T}^{\mathbf{I}}, \\
\mathbf{B}_{e}=\mathbf{T} \times \mathbf{B} \times \mathbf{G}^{\mathbf{I}}, \\
\mathbf{C}_{e}=\mathbf{G} \times \mathbf{C} \times \mathbf{T}^{\mathbf{I}},
\end{array}\right.
$$

where $\mathbf{w}_{e}=\mathbf{G} \times \mathbf{w}=\left[\begin{array}{llll}T_{u}^{*} & v_{3}^{*} & v_{3}^{*} & T_{w}^{*}\end{array}\right]$.

The controller $\mathbf{K}_{e}$ is contractible to the controller $\mathbf{K}$ if and only if there exist a matrix $\mathbf{G}$ such that

$$
\mathbf{K}_{e}=\mathbf{G} \times \mathbf{K} \times \mathbf{G}^{I},
$$

is achieved.

Proof 1

By replacing (8) and (9) in the closed loop overlapped system given by (17), we can find:

$$
\left\{\begin{array}{l}
\mathbf{T}^{I} \times \dot{\mathbf{X}}_{e}=[\mathbf{A}+\mathbf{B} \times \mathbf{K} \times \mathbf{C}] \mathbf{T}^{I} \times \mathbf{X}_{e}+\mathbf{B} \times \mathbf{G}^{\mathbf{I}} \times \mathbf{w}_{e}, \\
\mathbf{G}^{I} \times \mathbf{Y}_{e}=\mathbf{C} \times \mathbf{T}^{I} \times \mathbf{X}_{e},
\end{array}\right.
$$

which implies that:

$$
\left\{\begin{array}{l}
\dot{\mathbf{X}}_{e}=\mathbf{T} \times[\mathbf{A}+\mathbf{B} \times \mathbf{K} \times \mathbf{C}] \mathbf{T}^{I} \times \mathbf{X}_{e}+\mathbf{T} \times \mathbf{B} \times \mathbf{G}^{I} \times \mathbf{w}_{e} \\
\mathbf{Y}_{e}=\mathbf{G} \times \mathbf{C} \times \mathbf{T}^{I} \times \mathbf{X}_{e}
\end{array}\right.
$$

If we compare (22) with (18), we can notice that:

$$
\left\{\begin{array}{l}
\mathbf{A}_{e}+\mathbf{B}_{e} \times \mathbf{K}_{e} \times \mathbf{C}_{e}=\mathbf{T} \times[\mathbf{A}+\mathbf{B} \times \mathbf{K} \times \mathbf{C}] \mathbf{T}^{I}, \\
\mathbf{B}_{e}=\mathbf{T} \times \mathbf{B} \times \mathbf{G}^{I}, \\
\mathbf{C}_{e}=\mathbf{G} \times \mathbf{C} \times \mathbf{T}^{I} .
\end{array}\right.
$$

Taking into consideration (13) and (14), we can find that:

$$
\left\{\begin{array}{l}
\mathbf{B}_{e} \times \mathbf{K}_{e} \times \mathbf{C}_{e}=\mathbf{T} \times \mathbf{B} \times \mathbf{K} \times \mathbf{C} \times \mathbf{T}^{I}, \\
\mathbf{B}_{e}=\mathbf{T} \times \mathbf{B} \times \mathbf{G}^{I} \\
\mathbf{C}_{e}=\mathbf{G} \times \mathbf{C} \times \mathbf{T}^{I},
\end{array}\right.
$$

which implies that

$$
\begin{gathered}
\left(\mathbf{T} \times \mathbf{B} \times \mathbf{G}^{I}\right) \times \mathbf{K}_{e} \times\left(\mathbf{G} \times C \times \mathbf{T}^{I}\right) \mathbf{C}_{e}= \\
=\mathbf{T} \times \mathbf{B} \times \mathbf{K} \times \mathbf{C} \times \mathbf{T}^{I} .
\end{gathered}
$$

This gives us

$$
\mathbf{K}_{e}=\mathbf{G} \times \mathbf{K} \times \mathbf{G}^{I} .
$$

This means that expanded controller is contractible to the expanded non overlapped system if and only if there exists a matrix $\mathbf{G}$ such that $\mathbf{K}_{e}=\mathbf{G} \times \mathbf{K} \times \mathbf{G}^{I}$.

Proof 1 is completed.

If the control system (18) is an extension of the control system (17) and if (17) is stable (respectively asymptotically stable) then (18) is stable (respectively asymptotically stable) [16].

\section{Robust DeCEnTRALIZEd CONTROLler}

\section{A. Optimal System}

Consider the system (17), the goal is to find decentralized control law $\mathbf{u}=\mathbf{K y}_{x}$ shown in Fig. 1 that minimizes the cost function

$$
J=\int_{-\infty}^{+\infty}\left[\mathbf{X}^{T} \times \mathbf{Q} \times \mathbf{X}+\mathbf{U}^{T} \times \mathbf{R} \times \mathbf{U}\right] d t
$$

such that the closed loop system is asymptotically stable, $J^{*}$ is the desired cost function, the semi definite positive matrices $\mathbf{Q}$ and $\mathbf{R}$ are chosen using trial/error method [17]. The expanded problem of the expanded non overlapped system is given by (18), where the cost function is

$$
J_{e}=\int_{-\infty}^{+\infty}\left[\mathbf{X}_{e}^{T} \times \mathbf{Q}_{\mathbf{e}} \times \mathbf{X}_{e}+\mathbf{U}_{e}^{T} \times \mathbf{R}_{\mathbf{e}} \times \mathbf{U}_{e}\right] d t .
$$

We say that the pair $\left(S_{e}, J_{e}\right)$ includes the pair $(\mathrm{S}, \mathrm{J})$ if there is a pair of matrices $(\mathbf{T}, \mathbf{V})$ such that $\mathbf{T} \times \mathbf{V}=\mathbf{I}$, for any initial state $X_{0}$ of $(S, J)$ and any fixed input $U(t)$, we have:

$$
\left\{\begin{array}{l}
\mathbf{X}\left(t ; \mathbf{X}_{0}, \mathbf{U}\right)=\mathbf{T} \mathbf{X}_{e}\left(t ; \mathbf{V} \mathbf{X}_{0}, \mathbf{U}\right), \quad \forall t \geq 0 \\
J\left(\mathbf{X}_{0}, \mathbf{U}\right)=J_{e}\left(\mathbf{V} \mathbf{X}_{0}, \mathbf{U}\right) .
\end{array}\right.
$$

This condition is introduced in order to establish an optimal control problem of $\left(S_{e}, J_{e}\right)$ in the expanded space that provides a solution to the original problem. The matrices $\mathbf{Q}_{e}$ and $\mathbf{R}_{e}$ are semi definite positive, ant they can be calculated from (30):

$$
\left\{\begin{array}{l}
\mathbf{Q}_{e}=\mathbf{T} \times \mathbf{Q} \times \mathbf{T}^{I}, \\
\mathbf{R}_{e}=\mathbf{G} \times \mathbf{R} \times \mathbf{G}^{I} .
\end{array}\right.
$$

Proof 2

We have 


$$
\mathbf{X}_{e}\left(t ; \mathbf{x}_{0 e}, \mathbf{U}_{e}\right)=\mathbf{T} \times \mathbf{X}\left(t ; \mathbf{T} \mathbf{x}_{0}, \mathbf{T U}\right)
$$

or

$$
\mathbf{X}\left(t ; \mathbf{x}_{0}, \mathbf{U}\right)=\mathbf{T}^{I} \times \mathbf{X}_{e}\left(t ; \mathbf{T}^{I} \mathbf{x}_{0 e}, \mathbf{T}^{I} \mathbf{U}_{e}\right)
$$

If we replace (8) and (9) in (27), we find

$$
\begin{aligned}
J & =\int_{-\infty}^{+\infty}\left[\mathbf{X}_{e}^{T} \times \mathbf{T} \times \mathbf{Q} \times \mathbf{T}^{I} \times \mathbf{X}_{e}+\right. \\
& \left.+\mathbf{U}_{e}{ }^{T} \times \mathbf{G} \times \mathbf{R} \times \mathbf{G}^{I} \times \mathbf{U}_{e}\right] d t
\end{aligned}
$$

By comparing the last equation with (28), we can extract conditions given in (30). Thus, conditions for optimal decentralized non overlapped controller becomes:

$$
\left\{\begin{array}{l}
\mathbf{A}_{e}=\mathbf{T} \times \mathbf{A} \times \mathbf{T}^{I}, \\
\mathbf{B}_{e}=\mathbf{T} \times \mathbf{B} \times \mathbf{G}^{I}, \\
\mathbf{C}_{e}=\mathbf{G} \times \mathbf{C} \times \mathbf{G}^{I},
\end{array}\right.
$$

and:

$$
\left\{\begin{array}{l}
\mathbf{Q}_{e}=\mathbf{T} \times \mathbf{Q} \times \mathbf{T}^{I} \\
\mathbf{R}_{e}=\mathbf{G} \times \mathbf{R} \times \mathbf{G}^{I}
\end{array}\right.
$$

The last conditions are used to expand the overlapped system into a larger non overlapped system; it can be decomposed into two subsystems. To ensure that the dynamic of subsystems didn't change after decomposition, it is necessary to ensure that the interconnection terms are negligible [18]. By applying the condition given by (34) and (35) to the overlapped web winding system; we find that the expanded system is strongly connected, thus, interconnection terms can't be neglected. For this reason, we propose new conditions to ensure effectiveness of decomposition strategy.

\section{B. Decentralized Controller}

Consider the optimal problem for closed loop output feedback control of the overlapping web winding system before decomposition

$$
(S, J):\left\{\begin{array}{l}
\dot{\mathbf{X}}=[\mathbf{A}+\mathbf{B} \times \mathbf{K} \times \mathbf{C}] \mathbf{X}+\mathbf{B} \times \mathbf{w} \\
\mathbf{Y}=\mathbf{C} \times \mathbf{X} \\
J=\int_{-\infty}^{+\infty}\left[\mathbf{X}^{T} \times \mathbf{Q} \times \mathbf{X}+\mathbf{U}^{T} \times \mathbf{R} \times \mathbf{U}\right] d t
\end{array}\right.
$$

The expanded problem is

$$
\left(S_{e}, J_{e}\right):\left\{\begin{array}{l}
\dot{\mathbf{X}}_{e}=\left[\mathbf{A}_{e}+\mathbf{B}_{e} \times \mathbf{K}_{e} \times \mathbf{C}_{e}\right] \mathbf{X}_{e}+\mathbf{B}_{e} \times \mathbf{w}_{e}, \\
\mathbf{Y}_{e}=\mathbf{C}_{e} \times \mathbf{X}_{e}, \\
J_{e}=\int_{-\infty}^{+\infty}\left[\mathbf{X}_{e}^{T} \times \mathbf{Q}_{e} \times \mathbf{X}_{e}+\mathbf{U}_{e}^{T} \times \mathbf{R}_{e} \times \mathbf{U}_{e}\right] d t .
\end{array}\right.
$$

\section{Theorem}

The system $\left(S_{e}, J_{e}\right)$ is a restriction (expansion) of the system $(S, J)$ to $\operatorname{Im} \mathbf{T}$ if and only if there exist matrices $\mathbf{A}_{e q}, \mathbf{B}_{e q}, \mathbf{C}_{e q}, \mathbf{Q}_{e q}$ and $\mathbf{R}_{e q}$ such that:

$$
\left\{\begin{array}{l}
\mathbf{A}_{e}=\mathbf{T} \times \mathbf{A} \times \mathbf{T}^{I}+\mathbf{A}_{e q} \\
\mathbf{B}_{e}=\mathbf{T} \times \mathbf{B} \times \mathbf{G}^{I}+\mathbf{B}_{e q} \\
\mathbf{Q}_{e}=\mathbf{T} \times \mathbf{Q} \times \mathbf{T}^{I}+\mathbf{Q}_{e q} \\
\mathbf{R}_{e}=\mathbf{G} \times \mathbf{R} \times \mathbf{G}^{I}+\mathbf{R}_{e q}
\end{array}\right.
$$

and:

$$
\left\{\begin{array}{l}
\mathbf{A}_{e q} \times \mathbf{T}=0, \\
\mathbf{B}_{e q}=0, \\
\mathbf{C}_{e q}=0, \\
\mathbf{T}^{I} \times \mathbf{Q}_{e q} \times \mathbf{T}=0, \\
\mathbf{G}^{I} \times \mathbf{R}_{e q} \times \mathbf{G}=0 .
\end{array}\right.
$$

Proof 3

We establish only conditions for restriction; if we put $\mathbf{A}_{e c}=\mathbf{A}_{e}+\mathbf{B}_{e} \times \mathbf{K}_{e} \times \mathbf{C}_{e}$ and $\mathbf{A}_{c}=\mathbf{A}+\mathbf{B} \times \mathbf{K} \times \mathbf{C}, \quad$ so systems given in (17) and (18) become $\dot{\mathbf{X}}_{c}=\mathbf{A}_{c} \times \mathbf{X}_{c}+\mathbf{B}_{c} \times \mathbf{w}_{c}$ and $\dot{\mathbf{X}}_{e c}=\mathbf{A}_{e c} \times \mathbf{X}_{e c}+\mathbf{B}_{e c} \times \mathbf{w}_{e c}$, this implies that the solution of the original system is

$$
\mathbf{X}_{c}(t)=e^{\mathbf{A}_{c} \cdot t} \times \mathbf{X}_{0}+\int_{0}^{t} e^{\mathbf{A}_{c} \times(t-\tau)} \times \mathbf{B} \times \mathbf{w}(\tau) d \tau,
$$

and for the expanded system is

$\mathbf{X}_{e c}(t)=e^{\mathbf{A}_{e c} \times t} \times \mathbf{X}_{e 0}+\int_{0}^{t} e^{\mathbf{A}_{e c} \times(t-\tau)} \times \mathbf{B}_{e} \times \mathbf{w}_{e}(\tau) d \tau$.

From one side, we have

$$
\begin{gathered}
\mathbf{X}_{e}\left(t ; \mathbf{T} \times \mathbf{X}_{0}, \mathbf{T} \times \mathbf{U}\right)=\mathbf{T} \times \mathbf{X}\left(t ; \mathbf{X}_{0}, \mathbf{U}\right)= \\
=\mathbf{T} \times e^{\mathbf{A}_{c} \times t} \times \mathbf{X}_{0}+\mathbf{T} \times \int_{0}^{t} e^{\mathbf{A}_{c} \times(t-\tau)} \times \mathbf{B} \times \mathbf{w}(\tau) d \tau .
\end{gathered}
$$

From the other side, we have

$$
\begin{gathered}
\mathbf{X}_{e c}\left(t ; \mathbf{T} \times \mathbf{X}_{0}, \mathbf{T} \times \mathbf{U}\right)= \\
=e^{\mathbf{A}_{e c} \times t} \times \mathbf{X}_{0 e}+\int_{0}^{t} e^{\mathbf{A}_{e c} \times(t-\tau)} \times \mathbf{B}_{e} \times \mathbf{w}_{e}(\tau) d \tau \times \mathbf{X}_{e} .
\end{gathered}
$$

If we suppose that:

$$
\left\{\begin{array}{l}
\mathbf{A}_{e c}=\mathbf{T} \times \mathbf{A}_{c} \times \mathbf{T}^{I}+\mathbf{A}_{e q c} \\
\mathbf{B}_{e c}=\mathbf{T} \times \mathbf{B}_{c} \times \mathbf{G}^{I}+\mathbf{B}_{e q c} \\
\mathbf{w}_{e}(\tau)=\mathbf{G} \times \mathbf{w}(\tau) \\
\mathbf{X}_{0 e}=\mathbf{T} \times \mathbf{X}_{0}
\end{array}\right.
$$


By replacing the last values in (41), we find

$$
\begin{gathered}
\mathbf{X}_{e c}\left(t ; \mathbf{T} \times \mathbf{X}_{0}, \mathbf{T} \times \mathbf{U}\right)=e^{\left[\mathbf{T} \times \mathbf{A}_{c} \times \mathbf{T}^{I}+\mathbf{A}_{e q c}\right] \times t} \times \mathbf{T} \times \mathbf{X}_{0 e}+ \\
+\int_{0}^{t} e^{\left[\mathbf{T} \times \mathbf{A}_{c} \times \mathbf{T}^{I}+\mathbf{A}_{e q c}\right] \times(t-\tau)} \times \\
\times\left[\mathbf{T} \times \mathbf{B}_{c} \times \mathbf{G}^{I}+\mathbf{B}_{e q c}\right] \times \mathbf{G} \times \mathbf{w}(\tau) d \tau .
\end{gathered}
$$

If we compare (45) with (42), we can establish that:

$$
\left\{\begin{array}{l}
\mathbf{T} \times e^{\mathbf{A}_{e c} \times t}=e^{\left[\mathbf{T} \times \mathbf{A}_{c} \times \mathbf{T}^{I}+\mathbf{A}_{e q c}\right] \times t} \times \mathbf{T}, \\
\mathbf{T} \times \int_{0}^{t} e^{\mathbf{A}_{e c} \times(t-\tau)} \times \mathbf{B}_{e} \times \mathbf{w}_{e}(\tau) d \tau, \\
t \int_{0}^{t} e^{\left[\mathbf{T} \times \mathbf{A}_{c} \times \mathbf{T}^{I}+\mathbf{A}_{e q c}\right] \times(t-\tau)} \times\left[\mathbf{T} \times \mathbf{B}_{c} \times \mathbf{G}^{I}+\mathbf{B}_{e q c}\right] \times \\
\times \mathbf{G} \times \mathbf{w}(\tau) d \tau .
\end{array}\right.
$$

From the first term of (46), we have

$$
\begin{aligned}
& e^{\mathbf{A}_{e c} \times t}=\mathbf{T}^{I} \times e^{\left[\mathbf{T} \times \mathbf{A}_{c} \times \mathbf{T}^{I}+\mathbf{A}_{e q c}\right] \times t} \times \mathbf{T} \Rightarrow \\
& \Rightarrow \mathbf{A}_{e c} \times t=\mathbf{T} \times \mathbf{A}_{c} \times \mathbf{T}^{I} \times t+\mathbf{A}_{e q c} \times \mathbf{T} \times t .
\end{aligned}
$$

Which mean that $\mathbf{A}_{e q c} \times \mathbf{T}=0$ which completes proof for the first part of (38) and (39).

For the second part of (46)

$$
\begin{gathered}
\mathbf{T} \times \int_{0}^{t} e^{\mathbf{A}_{e c} \times(t-\tau)} \times \mathbf{B}_{e} \times \mathbf{w}_{e}(\tau) d \tau= \\
=\int_{0}^{t} e^{\left[\mathbf{T} \times \mathbf{A}_{c} \times \mathbf{T}^{I}+\mathbf{A}_{e q c}\right] \times(t-\tau)} \times \\
\times\left[\mathbf{T} \times \mathbf{B}_{c} \times \mathbf{G}^{I}+\mathbf{B}_{e q c}\right] \times \mathbf{G} \times \mathbf{w}(\tau) d \tau .
\end{gathered}
$$

To prove this part, we take Taylor power series of the exponentials as follows

$$
e^{\mathbf{A}_{e c} \times(t-\tau)} \simeq e^{\mathbf{A}_{e c} \times t}\left[\mathbf{I}-\tau \times \mathbf{A}_{e c}\right],
$$

and

$$
\begin{gathered}
e^{\left[\mathbf{T} \times \mathbf{A}_{c} \times \mathbf{T}^{I}+\mathbf{A}_{e q c}\right] \times(t-\tau)} \simeq e^{\left[\mathbf{T} \times \mathbf{A}_{c} \times \mathbf{T}^{I}+\mathbf{A}_{e q c}\right] \times t} \times \\
\times\left[\mathbf{I}-\tau\left(\mathbf{T} \times \mathbf{A}_{c} \times \mathbf{T}^{I}+\mathbf{A}_{e q c}\right)\right] .
\end{gathered}
$$

Equation (48) becomes

$$
\begin{gathered}
\mathbf{T} \times e^{\mathbf{A}_{e c} \times t} \int_{0}^{t}\left[\mathbf{I}-\tau \times \mathbf{A}_{e c}\right] \times \mathbf{B}_{e c} \times \mathbf{w}_{e}(\tau) d \tau= \\
=\mathbf{T} \times e^{\left[\mathbf{T} \times \mathbf{A}_{\mathbf{c}} \times \mathbf{T}^{I}+\mathbf{A}_{e q c}\right] \times t} \times
\end{gathered}
$$

$$
\begin{aligned}
& \quad \times \int_{0}^{t}\left[I-\tau\left(\mathbf{T} \times \mathbf{A}_{c} \times \mathbf{T}^{I}+\mathbf{A}_{e q c}\right)\right] \times \\
& \times\left[\mathbf{B}_{c} \times \mathbf{G}^{I}+\mathbf{B}_{e q c}\right] \times \mathbf{G} \times \mathbf{w}(\tau) d \tau .
\end{aligned}
$$

The last equality is true if and only if $\mathbf{A}_{e c}=\mathbf{T} \times \mathbf{A}_{c} \times \mathbf{T}^{I}+\mathbf{A}_{e q c} ; \quad \mathbf{A}_{e q c}$ is chosen such that $\mathbf{A}_{e q c} \times \mathbf{T}=0, \quad \mathbf{B}_{e c}=\mathbf{T} \times \mathbf{B}_{c} \times \mathbf{G}^{I} ; \quad \mathbf{B}_{e q c}=0$, $\mathbf{w}_{e}(\tau)=\mathbf{G} \times \mathbf{w}(\tau)[19]$.

The conditions for $\mathbf{Q}_{e q c}$ and $\mathbf{R}_{e q c}$ are demonstrated by following the same method; we replace (8) and (9) in (27), we find that $\mathbf{Q}_{e c}=\mathbf{T} \times \mathbf{Q} \times \mathbf{T}^{I}$ and $\mathbf{R}_{e c}=\mathbf{G} \times \mathbf{R} \times \mathbf{G}^{I}$ [20].

To guarantee the weakly coupling of the expanded web winding system, we proposed complementary matrices $\mathbf{Q}_{e q c}$ and $\mathbf{R}_{e q c}$ such that:

$$
\left\{\begin{array}{l}
\mathbf{Q}_{e c}=\mathbf{T} \times \mathbf{Q} \times \mathbf{T}^{I}+\mathbf{Q}_{e q c}, \\
\mathbf{R}_{e c}=\mathbf{G} \times \mathbf{R} \times \mathbf{G}^{I}+R_{e q c} .
\end{array}\right.
$$

Using (27), (29) and (52), we can find that:

$$
\begin{gathered}
J=J_{e}=\int_{-\infty}^{+\infty}\left[\mathbf{X}^{T} \times \mathbf{Q} \times \mathbf{X}+\mathbf{U}^{T} \times \mathbf{R} \times \mathbf{U}\right] d t= \\
=\int_{-\infty}^{+\infty}\left[\mathbf{X}_{e}^{T} \times \mathbf{Q}_{\mathbf{e}} \times \mathbf{X}_{e}+\mathbf{U}_{e}^{T} \times \mathbf{R}_{\mathbf{e}} \times \mathbf{U}_{e}\right] d t, \\
J=\int_{-\infty}^{+\infty}\left[\mathbf{X}^{T} \times \mathbf{Q} \times \mathbf{X}+\mathbf{X}^{T} \times \mathbf{T}^{I} \times \mathbf{Q}_{\mathbf{e q c}} \times \mathbf{T} \times \mathbf{X}+\right. \\
\left.+\mathbf{U}^{T} \times \mathbf{R} \times \mathbf{U}+\mathbf{U}^{T} \times \mathbf{G}^{I} \times \mathbf{R}_{e q c} \times \mathbf{G} \times \mathbf{U}\right] d t .
\end{gathered}
$$

The last equality is true if and only if:

$\left\{\begin{array}{l}\mathbf{X}^{T} \times \mathbf{T}^{I} \times \mathbf{Q}_{e q c} \times \mathbf{T} \times \mathbf{X}=0, \\ \mathbf{U}^{T} \times \mathbf{G}^{I} \times \mathbf{R}_{e q c} \times \mathbf{G} \times \mathbf{U}=0,\end{array} \Rightarrow\left\{\begin{array}{l}\mathbf{T}^{I} \times \mathbf{Q}_{e q c} \times \mathbf{T}=0, \\ \mathbf{G}^{I} \times \mathbf{R}_{e q c} \times \mathbf{G}=0 .\end{array}\right.\right.$

The requirement $\mathbf{Y}\left[\mathbf{X}_{e c}(t)=\mathbf{Y}\left[\mathbf{X}_{c}(t)\right]\right]$ that is $\mathbf{C}_{e c}=\mathbf{G} \times \mathbf{C}_{c} \times \mathbf{G}^{I}+\mathbf{C}_{e c q}$ is demonstrated as follows

$$
\mathbf{Y}_{e c}=\mathbf{C}_{e c} \times \mathbf{X}_{e c}=\left[\mathbf{G} \times \mathbf{C}_{c} \times \mathbf{G}^{I}+\mathbf{C}_{e c q}\right] \times \mathbf{T} \times \mathbf{X}_{c} .
$$

This implies that

$$
\mathbf{C}_{e c q} \times \mathbf{T}=0 \Rightarrow \mathbf{C}_{e c q} \times \mathbf{X}_{e}(t)=0 \Rightarrow \mathbf{C}_{e c q} \times \mathbf{T} \times \mathbf{X}(t)=0
$$

The change of basis $\mathbf{X}_{e}=\mathbf{T} \times \mathbf{X}$ yields to an equivalent representation. The new expanded system is weakly coupled and can be decomposed into two subsystems (Fig. 3); they can be controlled separately. The representation of first subsystem is 


$$
\bar{S}_{1}:\left\{\begin{array}{l}
\dot{\mathbf{X}}_{1}=\left[\begin{array}{cc}
\mathbf{A}_{c} & * \\
0 & *
\end{array}\right] \mathbf{X}_{e}+\left[\begin{array}{c}
\mathbf{B} \\
0
\end{array}\right] \times \mathbf{w}_{e}, \\
\mathbf{Y}_{1}=\left[\begin{array}{ll}
\mathbf{C} & 0
\end{array}\right] \times \overline{\mathbf{X}},
\end{array}\right.
$$

and the representation of subsystem two is

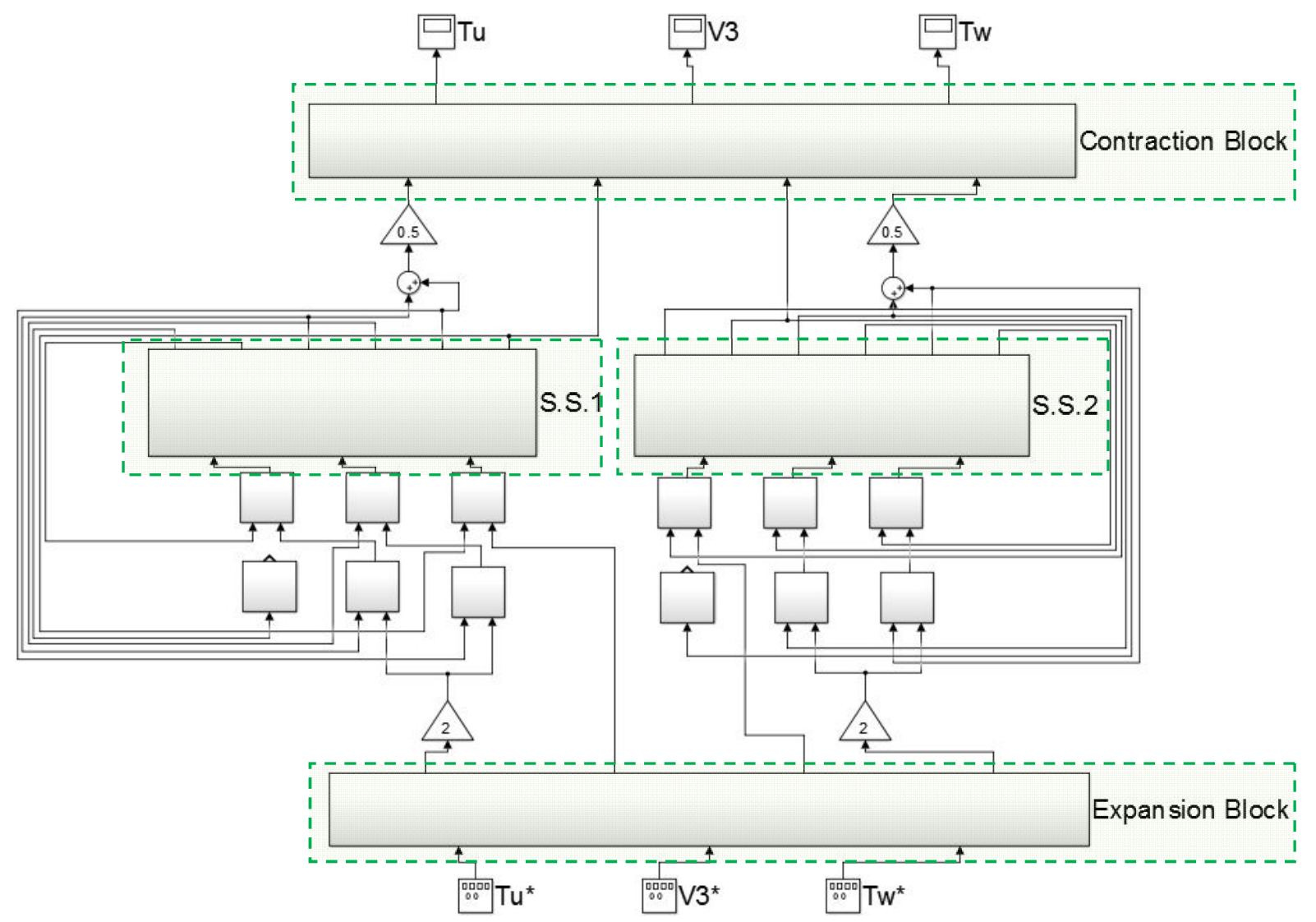

Fig. 3. Decentralized output feedback controller of decomposed web winding system.

We note that aggregation and restriction are mutually exclusive conditions; the aggregation specifies the behaviour of projection of the state trajectory of $S_{e}$ for arbitrary initial conditions $X_{e}$, whereas, the system $S_{e}$ includes all the information concerning the behaviour of $S$.

Using the proposed theorem, we generate an optimal output feedback control law of the form $u_{i}=K_{i} y_{i} ; i=1,2$ for each subsystem. By respecting the necessary and sufficient conditions of optimality [22], the developed algorithm is based on solving (38):

$$
\left\{\begin{array}{l}
\mathbf{A}_{c i}^{T} \mathbf{P}_{i}+\mathbf{A}_{c i} \phi_{i}+\mathbf{Q}_{i}+\mathbf{C}_{i}^{T} \mathbf{K}_{i}^{T} \mathbf{R}_{i} \mathbf{K}_{i} \mathbf{C}_{i}=0 \\
\mathbf{K}_{i}=-\mathbf{R}_{i}^{-1} \mathbf{B}_{i}^{T} \mathbf{P}_{i} \mathbf{L}_{i} \mathbf{C}_{i}^{T}\left(\mathbf{C}_{i} \mathbf{L}_{i} \mathbf{C}_{i}^{T}\right)^{-1}, \\
\mathbf{A}_{c i} \mathbf{L}_{i}+\mathbf{L}_{i} \mathbf{A}_{c i}^{T}+\mathbf{X}_{0 i}=0
\end{array}\right.
$$

where $\mathbf{X}_{0 i}=\mathbf{x}_{0 i} \times \mathbf{x}_{0 i}^{T}$; we take $\mathbf{x}_{0 i}=\mathbf{I}$.

Equation (60) can be solved by following three steps; first, we calculate the value of $L_{i}$ from the third part of the equation using the initial states, this value allows us to find the gain matrix $K_{i}$ from the second part of (60). By solving the first equation in (60) we find $P_{i}$ that allows us to calculate the cost function value $J_{i}$ and compare it to the

$$
\bar{S}_{2}:\left\{\begin{array}{l}
\dot{\overline{\mathbf{X}}}_{2}=\left[\begin{array}{cc}
* & * \\
0 & \mathbf{A}_{c}
\end{array}\right] \mathbf{X}_{e}+\left[\begin{array}{c}
* \\
\mathbf{B}
\end{array}\right] \times \mathbf{w}_{e} \\
\mathbf{Y}_{2}=\left[\begin{array}{ll}
0 & \mathbf{C}
\end{array}\right] \times \overline{\mathbf{X}}
\end{array}\right.
$$

The proof of theorem is completed [21]. 
function values (for the obtained subsystems) calculated from (37) are equal to $J_{1 d}^{*}=2.1 \times 10^{2}$ and $J_{2 d}^{*}=1.9 \times 10^{2}$ respectively. The values confirms the improvement of robustness of the controller since it reduced error of unwinding motor by $49.5 \%$, and error of winding tension by $54.3 \%$, and error of velocity by $33.3 \%$. In addition to that, the cost function has been optimized by $32.8 \%$.

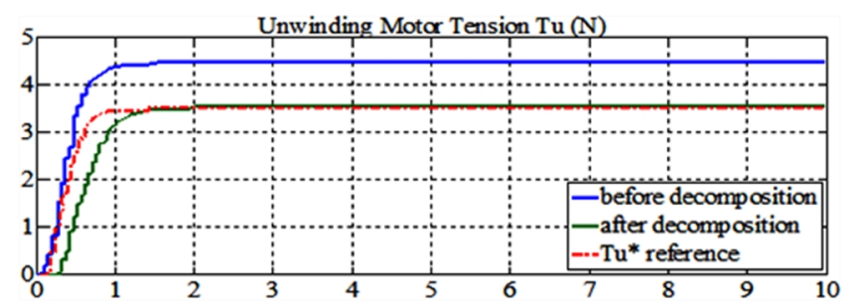

Fig. 4. Unwinding motor tension responses for web winding system before and after decomposition.

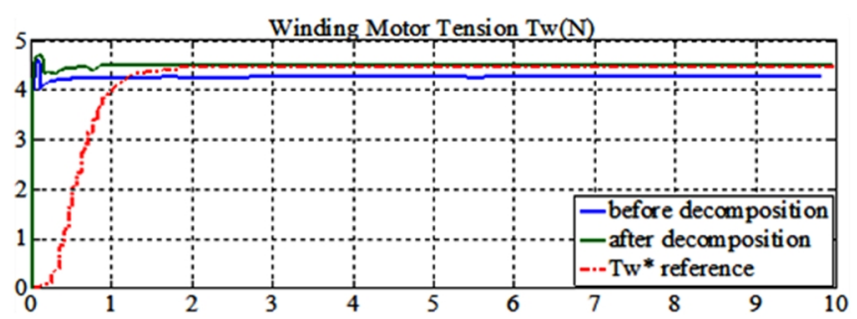

Fig. 5. Winding motor tension responses for web winding system before and after decomposition.



Fig. 6. Motor 3 velocity responses for web winding system before and after decomposition.

\section{B. Results for the Second Signal References}

As shown in Fig. 7-Fig. 9, the new desired signal reference are reached too, where

- Without decomposition: the dynamic of closed loop system follows the input reference signals for the unwinding tension with errors $e_{1}=13.7 \%$ (Fig. 7), and for the winding tension with error $e_{2}=10.9 \%$ (Fig. 8) and for the velocity input with error $e_{3}=3.9 \%$, the cost function value is found $J^{*}=5.31 \times 10^{3}$.

- With decomposition, the dynamic of closed loop system follows the unwinding tension with error $e_{1 d}=9.7 \%$ (Fig. 7), the winding tension with error $e_{2 d}=5.3 \%$ (Fig. 8), and the velocity input with error $e_{3 d}=1.7 \%$ where the cost values are $J_{1 d}^{*}=3.18 \times 10^{2}$ and $J_{2 d}^{*}=6.5 \times 10^{2}$. From the obtained results, the improvement of controller robustness is found, respectively, for unwinding motor $29.2 \%$, and for winding motor $51.4 \%$, and for third motor's velocity
$56.4 \%$. The energy consumed by the three motors of the system, represented by cost function, is reduced by $81.8 \%$.

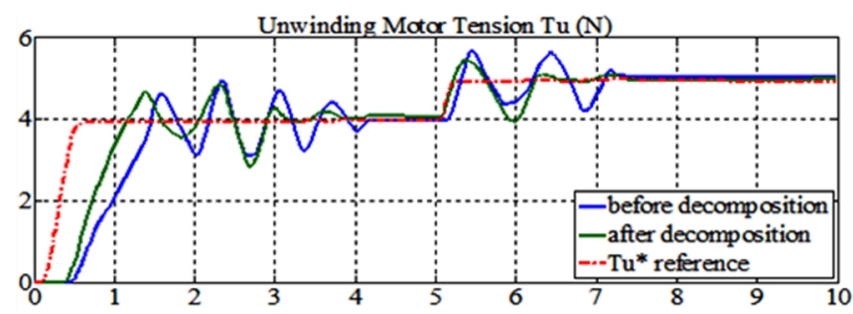

Fig. 7. Unwinding motor tension response for web winding system before and after decomposition.

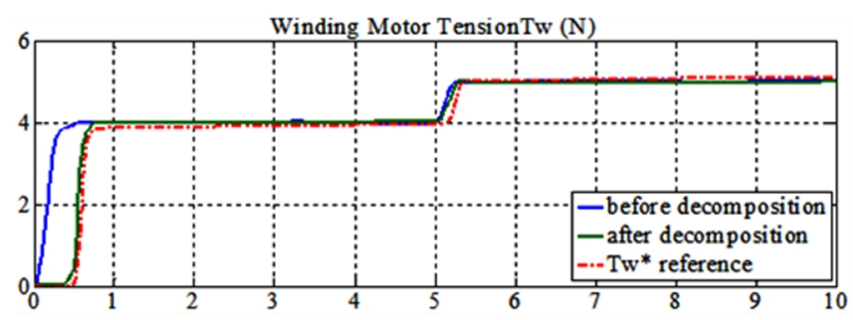

Fig. 8. Winding motor tension response for web winding system before and after decomposition.

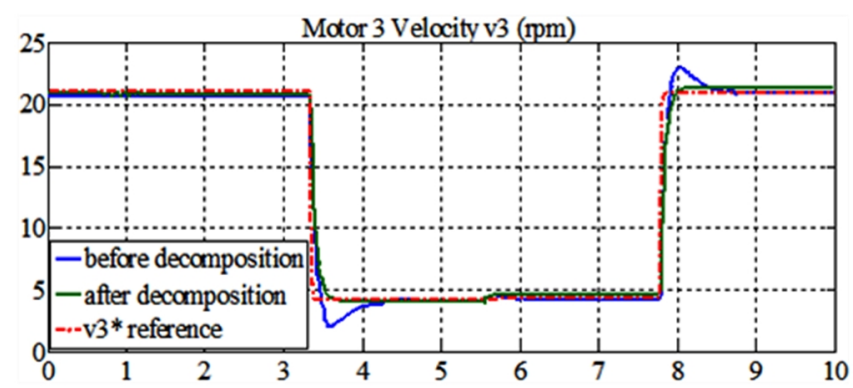

Fig. 9. Motor 3 velocity response for web winding system before and after decomposition.

\section{Results for Third Signal References}

- For unwinding motor: dynamic of the unwinding motor is similar to the third signal reference (Fig. 10) with cost function value equal to $J_{1 d}{ }^{*}=9.6574 \times 10^{3}$, this value has been reduced by $34.9 \%$ in comparison to the control system without implication any decomposition strategy and without reducing system complexity.

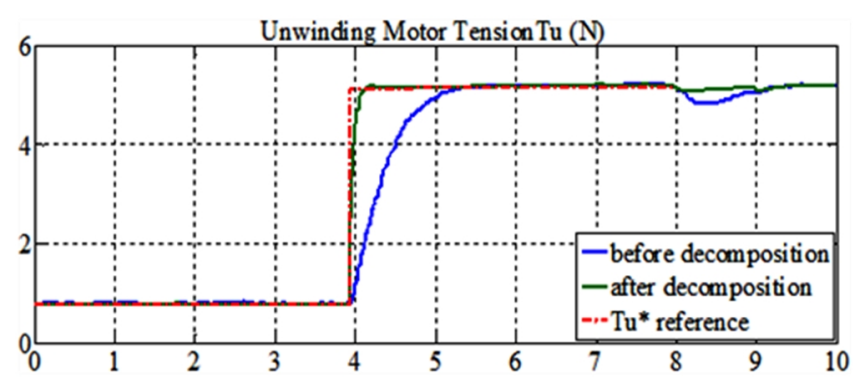

Fig. 10. Unwinding motor tension response for web winding system before and after decomposition.

- For winding motor: the winding motor response is the same as the desired signal (Fig. 11), the calculated value of the cost function is equal to $J_{2 d}{ }^{*}=0.82 \times 10^{3}$, this confirms the effectiveness of the decomposition strategy in reducing cost function of the winding motor by 
$42.5 \%$, and in simplifying the complexity of controller for industrial implementation.

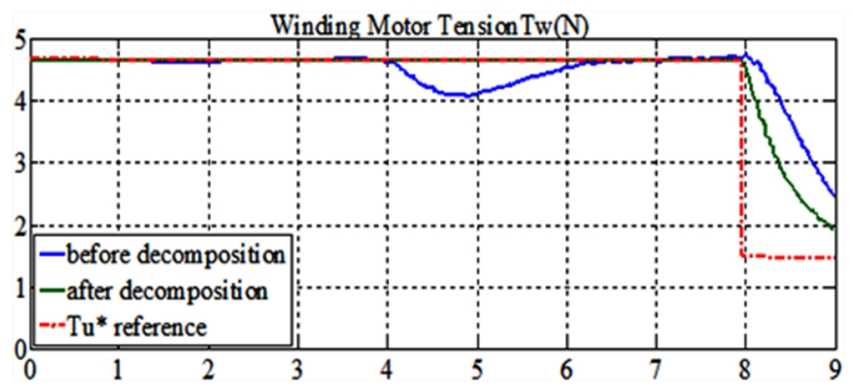

Fig. 11. Winding motor tension response for web winding system before and after decomposition.

\section{CONCLUSIONS}

In this paper, the overlapping decomposition strategy has been proposed to improve robustness of decentralized controller; the mathematical framework of the studied problematic has been introduced based on the inclusion/contraction principle. Moreover, optimality constraints have been included in order to incorporate the optimal control problem for large scale systems. The contractibility of contracted controller has been demonstrated through the cited theorem. Optimal decentralized dynamic output feedback controller has been designed with/without the proposed decomposition strategy and applied for three-motor web winding system. We found that the strategy increases efficiency of the designed controller, in addition to simplifying its implementation and reducing complexity of the system's model.

Furthermore, to improve the obtained results, we have integrated an optimization cost function so that the designed controller is optimized, and the cost function, which represents energy consumed by the three motors, is reduced. We noticed that the sum of cost values for decomposed subsystems is less than the cost function value of the original system $\left(J_{1}^{*}+J_{2}^{*} \prec J^{*}\right)$. To clarify the advantages of the proposed decomposition strategy, a comparison between results of control system with and without the strategy has been realized and discussed at the end of this paper.

Several future works can be realized based on this study, larger systems can be simplified especially where there are many overlapped regions with higher dimension systems. Fuzzy state-feedback controller can be used considered for future improvements.

\section{REFERENCES}

[1] N. I. Giannoccaro, G. Manieri, P. Martina, T. Sakamoto, "Genetic algorithm PI controller tuning of a multi-span web transport system based on overlapping decomposition", 11th Asian Control Conf (ASCC 2017), Gold Coast, QLD, Australia, 2017. DOI: 10.1109/ASCC.2017.8287306

[2] M. Z. Doghmane, M. Kidouche, H. Habbi, W. Lamraoui, "A new decomposition strategy approach applied for web winding system control optimization", IEEE 3rd Int. Conf. on Control , Engineering \& Information Technology (CEIT 2015), Tlemcen, Algeria, 2015. DOI: 10.1109/CEIT.2015.7233047.
[3] P. R Raul, P. R. Pagilla, "Design and implementation of adaptive PI control schemes for web tension control in roll-to-roll (R2R) manufacturing", ISA Trans., 2014. DOI: 10.1016/j.isatra.2014.11.020.

[4] H. Hou, X. Nian, J. Shang, S. Xu, Z. Peng, "Robust tension observer design for web-winding systems", $35^{\text {th }}$ Chinese Control Conf. (CCC 2016), Chengdu, China, 2016. DOI: 10.1109/ChiCC.2016.7553120.

[5] H. Hou, X. Nian, H. Xiong, Z. Wang, Z. Peng, "Robust decentralized coordinated control of a multimotor web-winding system", IEEE Trans. Control Systems Technology, vol. 24, no. 3, pp. 1495-1503, 2016. DOI: 10.1109/TCST.2015.2499705.

[6] Y-M. Fang, L. Lui, L. Jiang-xiong, R. Chang, "Compound control for speed and tension multivariable coupling system of reversible cold strip mill”, J. Cent. South Univ., vol. 22, pp. 529-538, 2015. DOI: 10.1007/s11771-015-2552-4.

[7] S. S. Stankovic, D. M. Stipanovic, M. S. Stankovic, "Decentralized overlapping tracking control", International Journal of General Systems, vol. 43, no. 3-4, pp. 282-293, 2014. DOI: 10.1080/03081079.2014.883713.

[8] H. Hou, X. Nian, S. Xu, M. Sun, H. Xiong, "Robust decentralized control for large-scale web-winding systems: A linear matrix inequality approach", Transactions of the Institute of Measurement and Control, pp. 1-12, 2016. DOI: 10.1177/0142331215622534.

[9] A. Aleksandrovas, A. Rodriguez, L. Svilainis, M. Angel de la Casa, A. Salazar, "Ultrasound-Based Density Estimation of Composites Using Water-Air Interface", Elektronika ir Elektrotechnika, vol. 22, no. 6, 2016. DOI: 28-32. DOI: 10.5755/j01.eie.22.6.17219.

[10] G. Feng, E. Li. Shengbo, Y. Zheng, K. Dongsuk, "Robust control of heterogeneous vehicular platoon with uncertain dynamic and communication delay", IET Intelligent Transport Systems, vol. 10, no. 7, pp. 503-513, 2016. DOI: 10.1049/iet-its.2015.0205.

[11] A. Ahamdi, M. Aldeen, "Robust overlapping load frequency output feedback control of multi-area interconnected power systems", International Journal of Electrical Power \& Energy Systems, vol. 89, pp. 156-172, 2017. DOI: 10.1016/j.ijepes.2016.12.015.

[12] M. Gutierrez Soto, H. Adeli, "Recent advances in control algorithms for smart structures and machines", Expert Systems, vol. 34, 2017. DOI: $10.1111 /$ exsy.12205.

[13] P. Benner, S. Gugercin, K. Willcox, "A survey of projection-based model reduction methods for parametric dynamical systems", SIAM Review, vol. 57, no. 4, pp. 483-531, 2015. DOI: $10.1137 / 130932715$.

[14] A. Iftar, "Inclusion, restriction and overlapping decompositions of Neutral systems with distributed time-delay", Science Direct IFAC, vol. 49, no. 4, pp. 073-078, 2016. DOI: 10.1016/j.ifacol.2016.05.013.

[15] A. Iftar, "Extension principle and controller design for systems with distributed time-delay", Kybernetika, vol. 53, no. 3, pp. 630-652, 2017. DOI: $10.14736 / \mathrm{kyb}-2017-4-0630$.

[16] F. Palacios-Quinonero, J. Rodellar, J. M. Rosell, "Sequential design of multi-overlapping controllers for longitudinal multi-overlapping systems", Journal Applied Mathematics and Computation, vol. 207, no. 3, pp. 1170-1183, 2010. DOI: 10.1016/j.amc.2010.01.130.

[17] L. Xiaohua, G. Jiawang, L. Yang, "Multi-overlapping decentralized control design based on dynamic output feedback for large scale interconnected systems with radial structure", IEEE 27th Chinese Conf. Control and Decision (CCDC 2015), Qingdao, China, 2015. DOI: 10.1109/CCDC.2015.7161869.

[18] S. Magdi Mahmoud, "Output feedback overlapping control design of interconnected systems with input saturation", Numerical Algebra, Control and Optimization, vol. 6, no. 2, pp. 127-151, 2016. DOI: 10.3934/naco.2016004.

[19] M. Kidouche, A. Charef, "A contructive methodology of Lyapunov function of composite systems with nonlinear interconnected term", International Journal of Robotics and Automation, vol. 21, no. 1, pp. 19-24, 2006. DOI: 10.2316/Journal.206.2006.1.206-2814.

[20] M. Kidouche, H. Habbi, "On Lyapunov stability of interconnected nonlinear system: recursive integration methodology", Nonlinear Dynamics, vol. 60, pp. 183-191, 2010. DOI: 10.1007/s11071-0099588-7.

[21] M. Kidouche, H. Habbi, M. Zelmat, "Stability of interconnected systems under structural perturbation: decomposition- aggregation approach", World Academy of Science, Engineering and Technology, vol. 37, pp. 351-355, 2008. DOI: 10.5281/zenodo.1060310.

[22] T. Zhang, X. Xia, "Decentralized adaptive fuzzy output feedback control of stochastic nonlinear large-scale systems with dynamic uncertainties", Information Sciences, 2015. DOI: 10.1016/j.ins.2015.04.002. 